Delft University of Technology

\title{
Observation of Falling Cosmic Objects Using GPS-Based FSR
}

Kabakchiev, H.; Behar, V.; Garvanov, I.; Kabakchieva, D.; Kabakchiev, K.; Rohling, H.; Kulpa, K.; Yarovoy, A.

DOI

10.23919/IRS.2018.8447936

Publication date

2018

Document Version

Final published version

Published in

2018 19th International Radar Symposium (IRS)

\section{Citation (APA)}

Kabakchiev, H., Behar, V., Garvanov, I., Kabakchieva, D., Kabakchiev, K., Rohling, H., Kulpa, K., \& Yarovoy, A. (2018). Observation of Falling Cosmic Objects Using GPS-Based FSR. In H. Rohling (Ed.), 201819 th International Radar Symposium (IRS) (pp. 1-9). [8447936] IEEE . https://doi.org/10.23919/IRS.2018.8447936

Important note

To cite this publication, please use the final published version (if applicable).

Please check the document version above.

\section{Copyright}

Other than for strictly personal use, it is not permitted to download, forward or distribute the text or part of it, without the consent of the author(s) and/or copyright holder(s), unless the work is under an open content license such as Creative Commons.

\section{Takedown policy}

Please contact us and provide details if you believe this document breaches copyrights.

We will remove access to the work immediately and investigate your claim. 
Green Open Access added to TU Delft Institutional Repository 'You share, we take care!' - Taverne project

\section{https://www.openaccess.nl/en/you-share-we-take-care}

Otherwise as indicated in the copyright section: the publisher is the copyright holder of this work and the author uses the Dutch legislation to make this work public. 


\title{
Observation of Falling Cosmic Objects Using GPS-Based FSR
}

\author{
H. Kabakchiev' ${ }^{1}$, V. Behar ${ }^{2}$, I. Garvanov ${ }^{3}$, D. Kabakchieva ${ }^{4}$, K. Kabakchiev ${ }^{5}$, H. \\ Rohling $^{6}$, K. Kulpa ${ }^{7}$, A. Yarovoy ${ }^{8}$
}

${ }^{1}$ Sofia University "St. Kliment Ohridski"

Sofia, BULGARIA

e-mail: ckabakchiev@fmi.uni-sofia.bg

${ }^{2}$ Institute of Information and Communication Technologies

Sofia, BULGARIA

e-mail: behar@bas.bg

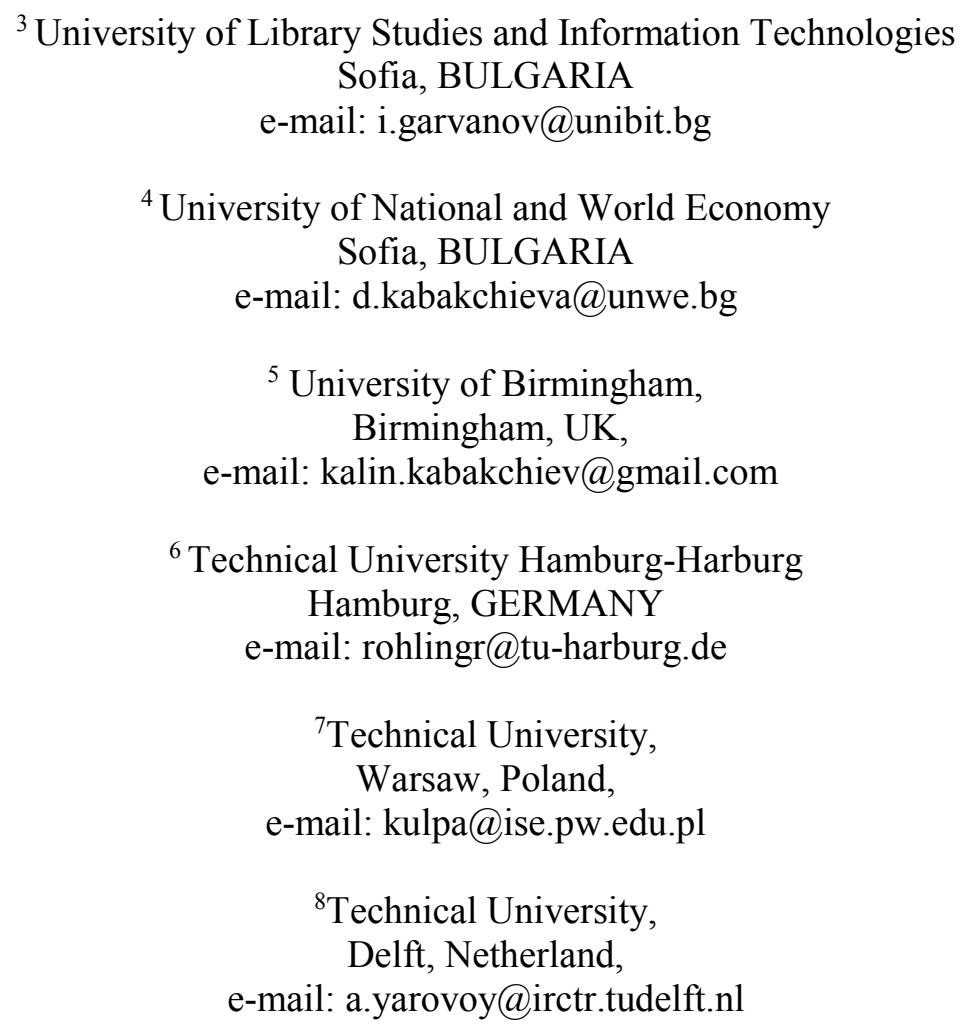

\begin{abstract}
In this paper, the possibility of GPS-based Forward Scatter Radar (GPS FSR), where satellites are exploited as sources of signals, to detect falling cosmic objects is examined. It is known that in FSR systems, the signal re-reflected by falling cosmic objects as masked by a much stronger direct signal. The goal of the article is to evaluate signalto-noise ratio (SNR) of these signals at the detector input, after the correlator and the integrator. The results obtained show that the difference in SNR between direct and rereflected signals is substantially dependent on the size of the falling object and the distance to it. The results obtained indicate what signal processing needs to be performed to separate the useful target signal from the direct signal.
\end{abstract}




\section{Introduction}

GPS-based Forward Scatter Radar (GPS FSR) is a type of passive radar where GPS satellites are sources of electromagnetic radiation. In GPS FSR, some air object can be detected when it crosses a narrow area near the baseline between a satellite and a GPS receiver (angular deviation from the baseline is in the range of $\pm 5^{0}$ ). In that case the target detection is based on the Forward Scatter (FS) effect, which appears when the wavelength of electromagnetic waves, incident on the target, is much less than the target's size $(D)$ and the distances to the target from both the receiver and the transmitter are much more than $4 D^{2} / \lambda$ ( $\lambda$ is the wavelength). Due to the FS effect, the Radar Cross Section (RCS) of air targets extremely increases and mainly depends on the area of the target's silhouette.

In last years, passive GPS-based radar systems (bistatic and FSR) became increasingly popular as an alternative to traditional radar systems. In $[1,2]$, the authors examine the idea to detect air targets using bistatic and forward scatter radar, which exploit GPS satellites as transmitters. In these articles they make the theoretical analysis of possibilities to detect air or sea targets in such radar systems and talk over on the eventual difficulties in the practical implementation of such systems. In their opinion, the main difficulty is related to the separation of the direct satellite signal from the signal scattered from the target. The usage of GPS L1 signal in FSR for air target detection is discussed in [3]. Some experimental results for air target detection using GPS L1-based FSR system are presented and discussed in [4]. A possible algorithm for air target detection using GPS L5-based FSR system is described in [6], and the detection probability characteristics are analytically calculated in [7] for low-flying and poorly manoeuvrable air targets in the urban interference environment. In these articles, the authors point out the potential of GPS L5 signal to increase SNR for target detection due to the increase in his radiated power. The articles [8 -14] discuss on the experimental measurements made by using GPS L1-based FSR system and the Software-Defined GPS receiver [5], which allows observing the GPS shadows of different ground objects.

This paper focuses at the very important problem of the Earth protection from unwanted incident cosmic objects. At present, there are two program systems (SCOUT and SENTRY) used for detection of Near-Earth Objects and designed by NASA [2]. Their main task is to detect $90 \%$ of asteroids with the diameter of $140 \mathrm{~m}$. and larger. Asteroids are big cosmic objects with the diameter of $140 \mathrm{~m}$. and larger. Meteoroids are small cosmic objects that are hard to find in space. The meteoroid's diameter varies from $0.1 \mathrm{~m}$. to $10 \mathrm{~m}$. Approaching the Earth, they (asteroid or meteoroid) enter into the atmosphere at an angle of about 45-46 degrees (Fig.1). Their average speed is about $20-30 \mathrm{~km} / \mathrm{s}$.

In most cases, meteoroids burn in the atmosphere, but sometimes parts of them (meteorites) reach the Earth's surface and pose a danger to humans and objects on Earth. The larger the meteoroids, the more dangerous they are for Earth's life. In this paper we analyse the power budget in SNR of GPS FSR receiver needed for observation of meteoroids and asteroids, which consists of a transmitter mounted on a satellite of GPS and a GPS receiver located on the Earth's surface (Fig. 1). For simplicity, the asteroid and meteorite will be called flying cosmic objects. In estimating this budget, we assume that a part of the trajectory of the meteorite or asteroid crosses or passes very close to the baseline of the system. The appearance of the FS effect at the radiation of the meteorite or asteroid by the GPS signal is possible only if the summed target distance to the satellite and to the receiver is commensurate with the distance of the baseline. The received mixture consists of two signals, the signal from the satellite and the signal reradiated by the meteoroid or asteroid. These signals cannot be spatially distinguishable due to the fact that both the GPS and the cosmic object have the same azimuth and elevation angle. The received useful signal re-emitted from the flying comic object is much weaker than the 
reference satellite signal. We also assume that during the observation of the cosmic objects in the FSR system, the satellite does not move, i.e. its velocity is zero.

The purpose of this study is to see the dynamics in the SNR of the two signals, the reference signal and the signal from a cosmic object, at the output of the GPS FSR receiver, depending on the distance to the cosmic object. That will also show the potential possibility for detection of cosmic objects depending on the distance to the object, with the required amount of noncoherent integration of GPS signals.

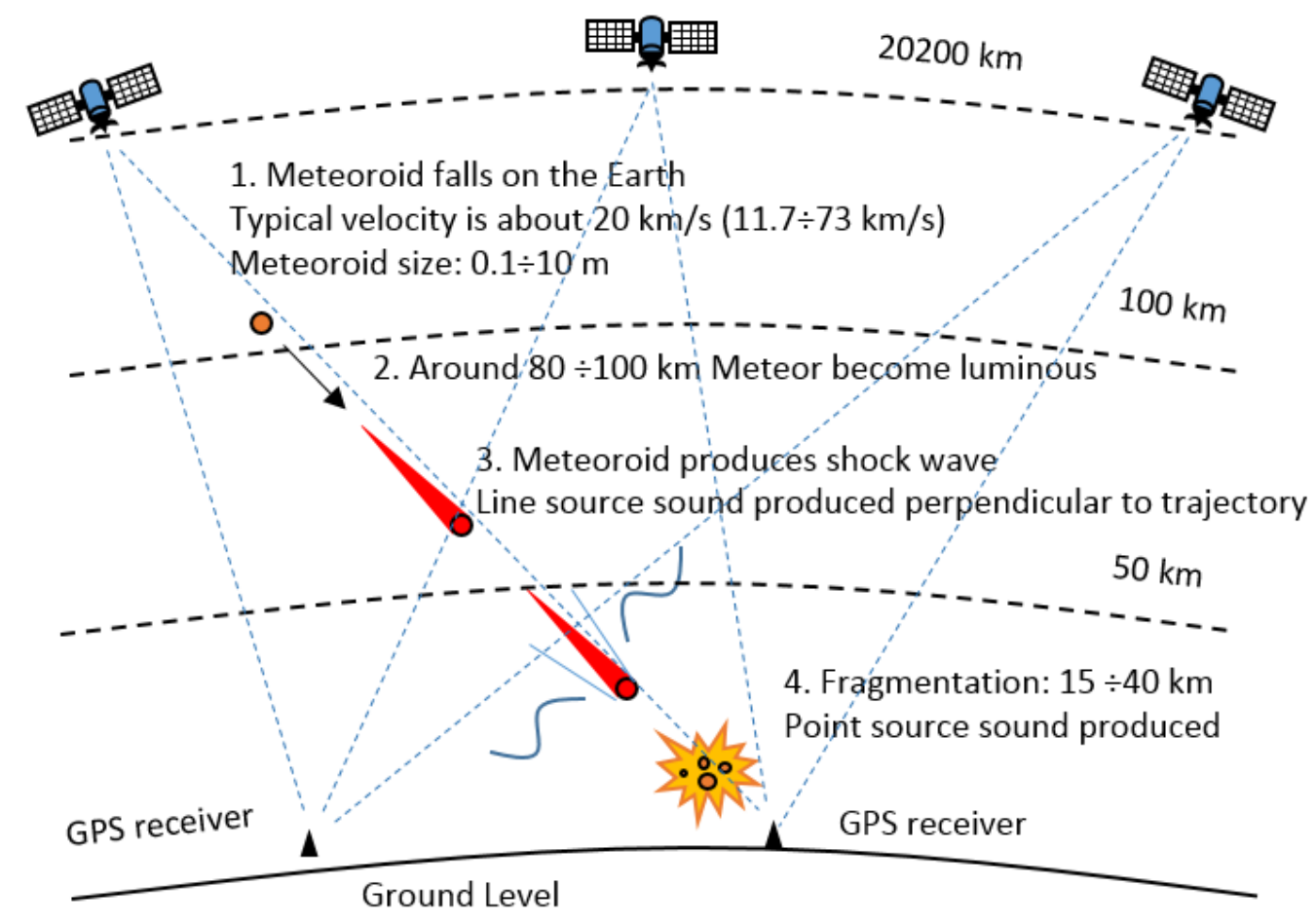

Figure 1. Entry of meteoroid into the earth's atmosphere

More concretely, we consider the GPS-based FSR system that uses the GPS L5 signal in order to increase maximally SNR detection of meteoroids. The results thus obtained will form the requirements for the type of processing algorithms, for detection of the useful signal.

\section{FSR Signal Processing}

The detection scheme considered in this section for GPS FSR system is shown on Figure 2.

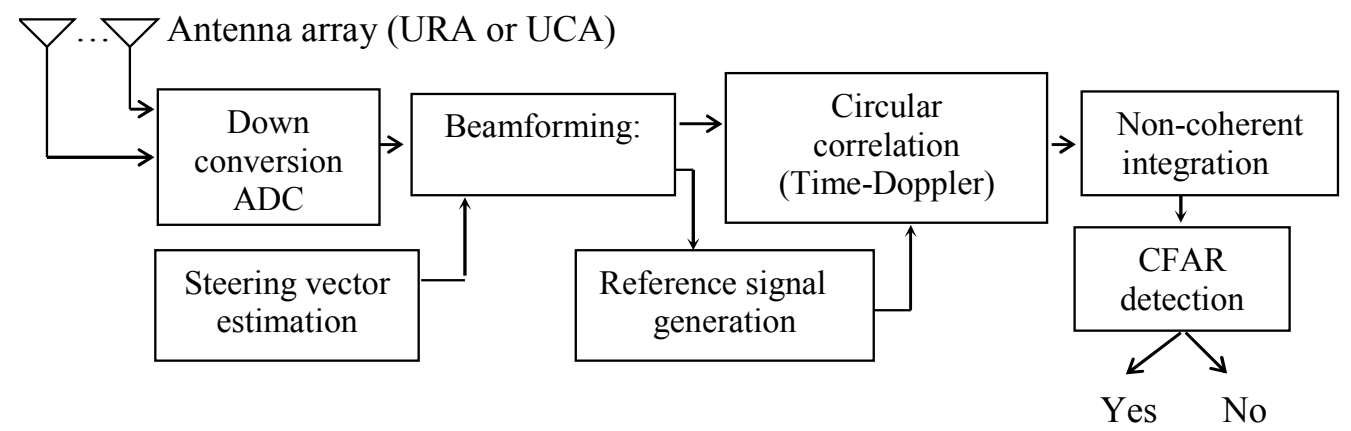

Figure 2. Signal processing scheme for GPS FSR 
The reference signal needed for cross-correlation is obtained by synchronization of the NH20 code in the reference channel. The synchronization process consists of two parts - acquisition and tracking. The coarse frequency estimate obtained using the acquisition algorithm is then passed to the tracking algorithm for fine Doppler estimation. The coherent processing is performed by correlating the reference signal with the frequency shifted target signal.

The above-mentioned processing algorithm of the GPS L5 signal provides the very good surface coverage on the ground for navigation, due to the much higher power of the L5 signal (by about $20 \mathrm{~dB}$ ) than of the L1 signal. In most cases, in the FSR system, the signal re-radiated from the cosmic object, is much weaker than the L5 signal (by 20-40 dB). Therefore, the task of the signal processing is the detection of a very weak signal from the cosmic object, which is masked by a strong L5 signal. This task can be solved using different algorithms. For example, one of them is to substantially increase the useful SNR at the output of the correlator by noncoherent accumulation. The amount of non-coherent accumulations must be sufficient to obtain a positive SNR that is enough to automatically detect the useful signal from the cosmic object. Naturally, such non-coherent accumulation does not change the ratio of the useful SNR of the signal from the cosmic object to the SNR of the direct signal from the GPS satellite.

To change this relationship between the two signals in favor of the useful one, we can use the approach of suppressing the reference signal, for example by suppressing it with a filter. This suppressive filter can be implemented in different ways. Then, the signal detector based on some CFAR (Constant False Alarm Rate) algorithm can be used to indicate detection of a cosmic object. This article does not deal with such algorithms for suppressing and autodetection.

\section{SNR of the Direct and Irradiated Signals at the Detector Input}

The power density $\left(S_{l}\right)$ at the output of an omnidirectional antenna near the Earth's surface can be determined as follows:

$$
S_{1}=P_{t} / A_{\text {omn }}, \text { where } A_{\text {omn }}=\lambda^{2} / 4 \pi
$$

In (1), $P_{\mathrm{t}}$ is the power of the GPS L5 signal measured near the Earth's surface (from $-153 \mathrm{dBw}$ to $-160 \mathrm{dBw}), A_{\text {omn }}$ is the effective area of the omnidirectional antenna, and $\lambda$ is the wavelength of the GPS L5 signal transmitted by a satellite. The power of the signal reradiated from a meteoroid with radar cross section (RCS) $\sigma$ in direction of the GPS receiver is:

$$
P_{m e t}=S_{1} \sigma=4 \pi P_{t} \sigma / \lambda^{2}
$$

The power density of the reradiated signal at the receiver antenna input $\left(S_{2}\right)$ is:

$$
S_{2}=\frac{P_{m e t}}{4 \pi R_{m e t}^{2}}=\frac{P_{t} \sigma}{\lambda^{2} R_{m e t}^{2}}
$$

The signal power at the output of the receiver antenna depends on the effective area of the receiver antenna, i.e. the antenna gain:

$$
P_{\text {rec }}=S_{2} A_{\text {omn }} G_{r}=\frac{P_{t} G_{r} \sigma}{4 \pi R_{\text {met }}^{2}}
$$

The noise level $N_{\mathrm{r}}$ at the output of the radio frequency (RF) front-end can be determined in terms of the equivalent noise temperature $(T)$ and the receiver bandwidth $(B)$ :

$$
N_{r}=k T B
$$

In (5), $k$ is the Boltzmann constant $(1.38 \times 10$ in units $(\mathrm{w} / \mathrm{K} / \mathrm{Hz})$. For a GPS L5 receiver, the frequency bandwidth is $20.46 \mathrm{MHz}$, and the noise level $N_{\mathrm{r}}$ in decibels is nearly $-131 \mathrm{dBw}$. Using (4) and (5), the SNR at the RF front-end output of the GPS receiver can be written as: 


$$
S N R_{\text {rec }}=\frac{P_{r e c}}{N_{r}}=\frac{P_{t} G_{r} \sigma}{4 \pi R_{m e t}^{2} N_{r}}
$$

The subsequent coherent signal processing includes circular cross-correlation. Therefore, the signal-to-noise ratio at the cross-correlator output is given by:

$$
S N R_{c o r}=S N R_{r e c} G_{S P}=\frac{P_{t} G_{r} \sigma G_{S P}}{4 \pi R_{m e t}^{2} N_{r}}
$$

In (7), $G_{S P}=B T_{Q 5}$ is the processing gain of the cross-correlator, and $T_{Q 5}$ is the period of the Q5 component of the GPS L5 signal. The signal to-noise ratio (7) could be improved by noncoherent integration over a detection time period $T_{\text {det. }}$. The maximal number of non-coherent samples is evaluated as:

$$
N=T_{\text {det }} / T_{Q 5}
$$

We assume that the observation time is $T_{\text {det }}=1-2$ seconds, taking into account the velocity of the falling cosmic object and the distance, which the object passes over that time. To achieve the FS effect, we assume that the cosmic object crosses the baseline at a very small deviation angle (1-2 degrees), or part of its trajectory is almost parallel to it (the deviation angle is almost zero).

After non-coherent integration, the final SNR of cosmic objects at the detector input is:

$$
S N R_{\mathrm{det}}=S N R_{c o r} N^{0.8}=\frac{P_{t} G_{r} \sigma G_{S P} N^{0.8}}{4 \pi R_{\text {met }}^{2} N_{r}}
$$

If the minimal value of SNR needed for signal detection with a given probability of detection is $S N R_{\mathrm{det} \text {,min, }}$ then the expression for calculation of the maximum meteoroid detection range is:

$$
R_{\text {met,max }}=\sqrt{\frac{P_{t} G_{r} \sigma G_{S P} N^{0.8}}{4 \pi N_{r} S N R_{\mathrm{det}, \text { min }}}}
$$

The FS effect is observed at distances $\left(R_{m e t}\right)$ of more than $4 D^{2} / \lambda$, i.e. in the area of Fraunhofer diffraction. In case of the FS effect the meteoroid RCS in (13) is calculated as:

$$
\sigma=\frac{4 \pi A_{m e t}^{2}}{\lambda^{2}}
$$

In (14) $A_{m e t}{ }^{2}$ is the silhouette area of a meteoroid. If we approximate the meteoroid as a sphere with a diameter $D$, then the RCS in (14) can be rewritten as:

$$
\sigma=\frac{\pi^{3} D^{4}}{4 \lambda^{2}}
$$

After non-coherent integration, the final SNR of the GPS signal at the detector input, is:

$$
S N R_{\text {direct }}=\frac{P_{t} G_{r} G_{S P} N^{0.8}}{N_{r}}
$$

The final signal-to-noise ratio of the mixed between GPS signal and signal of cosmic objects at the detector input, after non-coherent integration, is:

$$
S N R_{\text {full }}=S N R_{\text {direct }}+S N R_{t}=S N R_{\text {direct }}\left(1+\frac{\sigma_{t}}{4 \pi R^{2}}\right)
$$

The relationship below shows the difference in power or SNR between the received direct GPS signal and the re-radiated signal from a cosmic object.

$$
\frac{P_{\text {rec }}}{P_{\text {direct }}}=\frac{S N R_{\text {det }}}{S N R_{\text {direct }}}=\frac{\sigma_{t}}{4 \pi R^{2}}
$$




\section{Numerical Results for SNR at the Detector Input}

We assume that a large part of the sky is observed at the same time, i.e. several satellites are observed by several antennas with a large gain, and the signals from them and correspondingly from the falling cosmic objects are simultaneously recorded in one common receiver.

Then we have the option to choose that system "satellite - target - receiver" that best satisfies the conditions for the appearance of the FS effect. The below estimates of SNR for both signals, the direct signal and the signal from the cosmic object, are based on this hypothesis. Figure 3 shows the parameters of the FS effect: the zone of Fraunhofer diffraction and the angle of the shadow generated by the target.

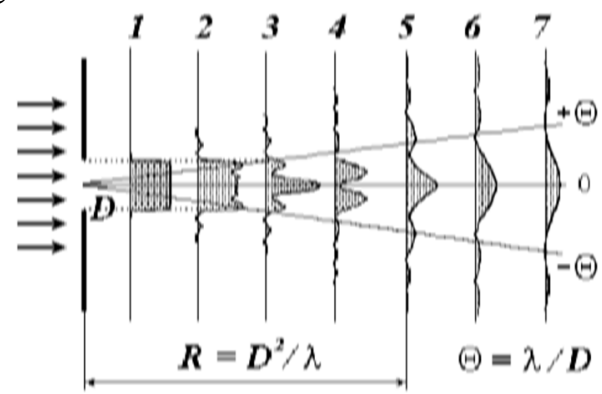

Figure 3. Diffraction of light passed through the circular aperture

In this section the analytical formulas obtained above are accompanied by numerical calculations of SNR at the detector input. We presented SNR of the signal from cosmic objects and SNR of the GPS signal at the detector input, after non-coherent integration, as a function of distance to cosmic objects. The results are obtained for three values of the number of noncoherent integrations of the signal: $N=1,10$ and 100. The values of SNR are calculated and plotted below for different diameters of a meteoroids $D=1,5$ and $10 \mathrm{~m}$, and asteroids $\mathrm{D}=150$, 300,500 and $800 \mathrm{~m}$. It is assumed that the meteoroids and asteroids move at velocity of $20 \mathrm{~km} / \mathrm{s}$. The numerical results are obtained with the antenna gain $G_{\mathrm{r}}=20 \mathrm{~dB}$.

The following parameters of the GPS L5 signal are used in calculations: carrier frequency $f_{0}=1176 \mathrm{MHz}(\lambda=0.2551 \mathrm{~m})$, frequency bandwidth $B=20.46 \mathrm{MHz}$, Q5 component period $T_{Q 5}=0.02 \mathrm{~s}$ (coherent processing interval), the power of the GPS L5 signal measured near the Earth's surface is $P_{\mathrm{t}}=-154 \mathrm{dBW}$. The FS effect is observed at distances $R_{m e t}>R_{F S}=D^{2} / \lambda$, i.e. in the area of Fraunhofer diffraction. According to the principle of Fraunhofer diffraction, the target is seen from the receiver at angle $\alpha$. Parameters $R_{F S}$ and $\alpha$ are presented in Table 1 according to the diameter $(D)$ of cosmic objects. It is easy to see that the larger the diameter of falling object, the narrower the angle of its shadow $\alpha=\lambda / \mathrm{D}$. The FS effect appears at a great distance to a cosmic object (from several kilometres to thousands of kilometres depending on the diameter of the object).

\begin{tabular}{|c|c|c|}
\hline $\begin{array}{c}\text { Meteoroid diameter (D) } \\
{[\mathrm{m}]}\end{array}$ & $\begin{array}{c}\text { Distance } R_{F S} \\
{[\mathrm{~km}]}\end{array}$ & $\begin{array}{c}\text { Angle } \alpha \\
{\left[{ }^{0}\right]}\end{array}$ \\
\hline 1 & 0.015 & 0.255 \\
\hline 5 & 0.392 & 0.051 \\
\hline 10 & 1.568 & 0.0255 \\
\hline 150 & 88,235 & 0.0017 \\
\hline 300 & 352,94 & 0.00085 \\
\hline 500 & 980,39 & 0.00051 \\
\hline 800 & 2509,80 & 0.00032 \\
\hline
\end{tabular}

Table 1. Forward scattering distance and angle as a function of meteoroid diameter 
Figure 4 and Figure 5 show the difference in SNR of the received direct signal and the reradiated FS signal from the cosmic objects, depending on different diameters of meteoroids. It can be seen that this diffrence in SNR is directly proportional to the FS radar cross section (FS RCS) of the cosmic object, and inversely proportional to the square of the distance to the object, and does not depend on the parameters of the GPS FSR system. These graphics are universal because they show the dependence of the difference in SNR between the direct and the redirected signal depending on the size of the falling objects and the distance to it, in FSR systems monitoring the space with different signal sources (satellites, pulsars, etc.).

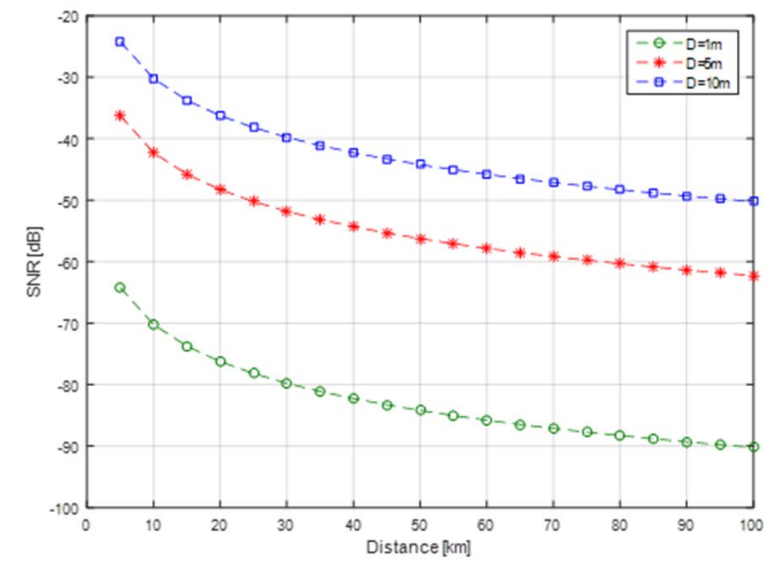

Figure 4. SNR of cosmic objects

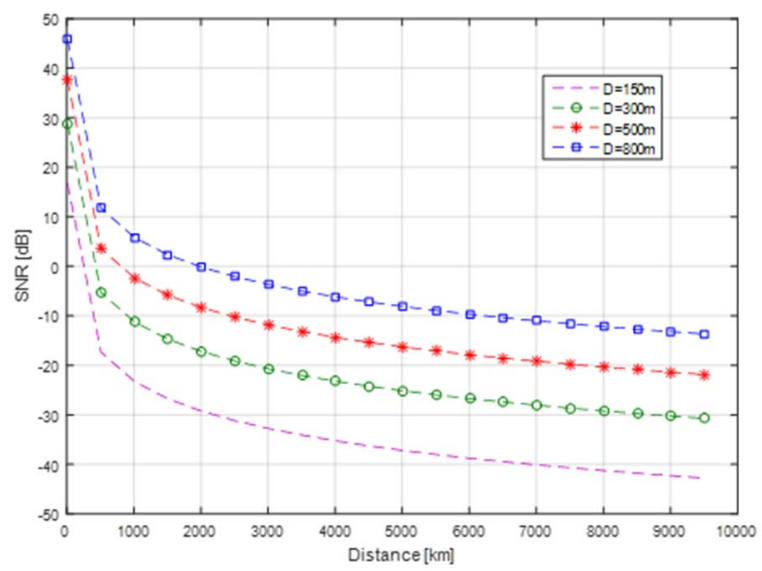

Figure 5. SNR of cosmic objects

The final SNR of cosmic objects and the GPS signal at the detector input, after non-coherent integration $(N=1,10$ и 100) is calculated and plotted below for different diameters of meteoroids: $D=1,5,10 \mathrm{~m}$, on Fig. 6,8 and 10 .

The same SNR for asteroids with diameters $D=150,300,500,800 \mathrm{~m}$, after non-coherent integration $(N=1,10$ и 100) is calculated and plotted below on Fig. 7, 9 and 11.

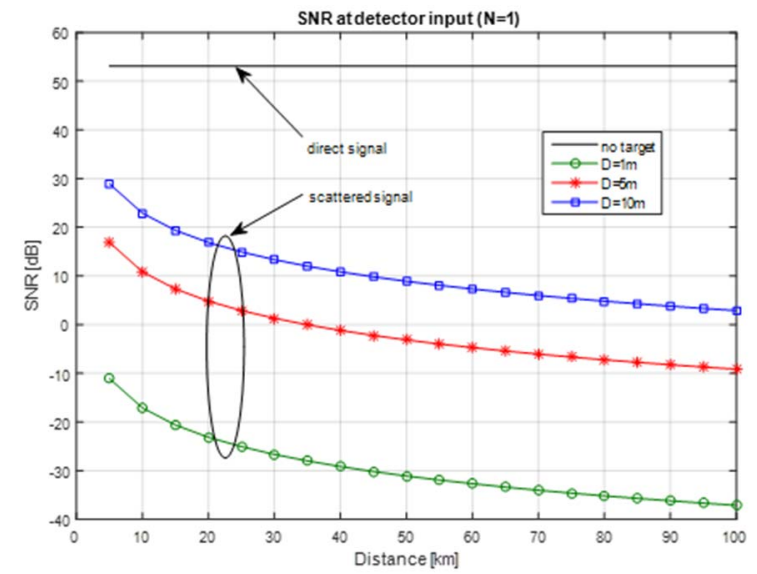

Figure 6. SNR of direct and scattered GPS signal

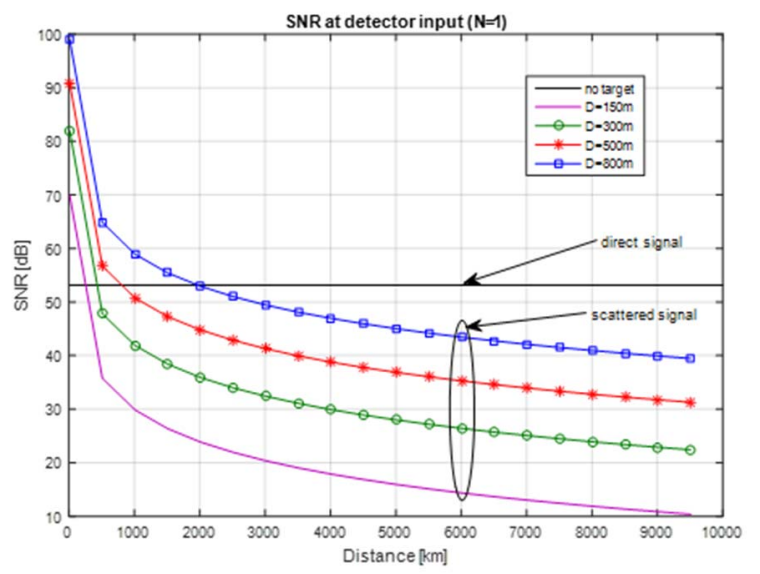

Figure 7. SNR of direct and scattered GPS signal

From the results in Fig. 6-11 follows that the direct GPS signal received by the receiver on the surface of the Earth has a constant magnitude and it is represented as a straight line. Naturally, the magnitude of the direct signal depends on the number of non-coherent integrations of the signal at the output of the correlator. 


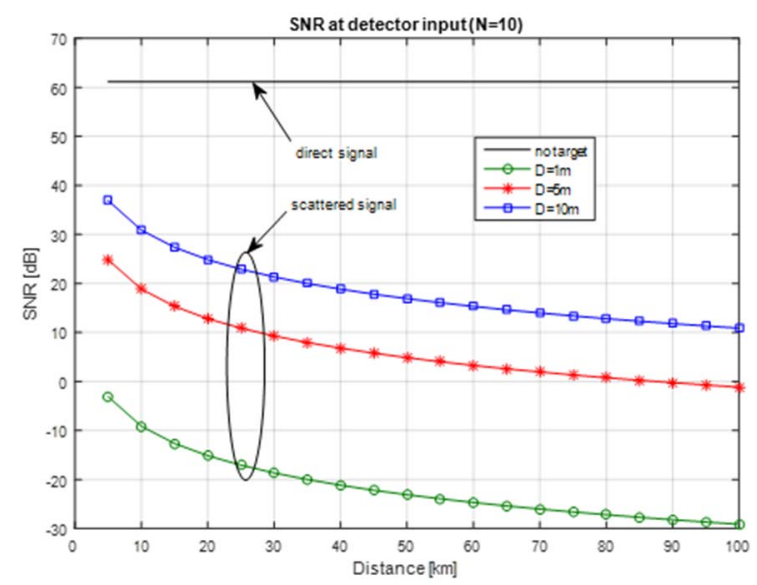

Figure 8. SNR of direct and scattered GPS signal

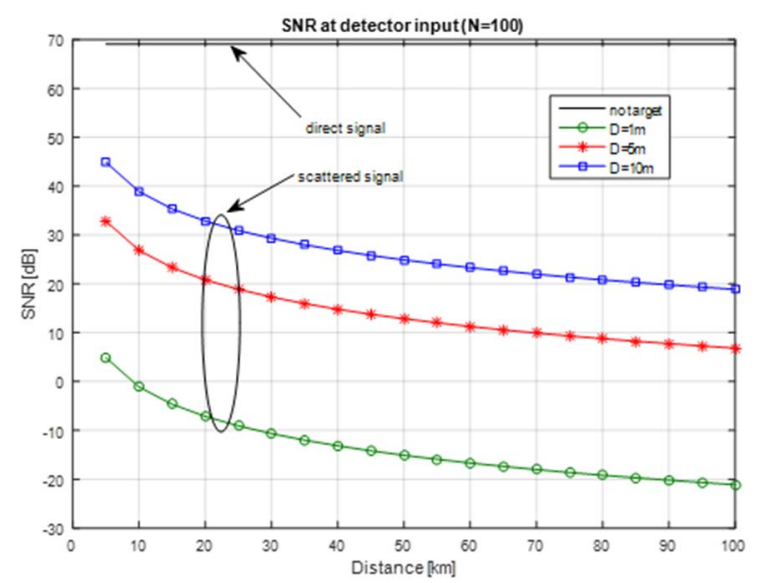

Figure 10. SNR of direct and scattered GPS signal

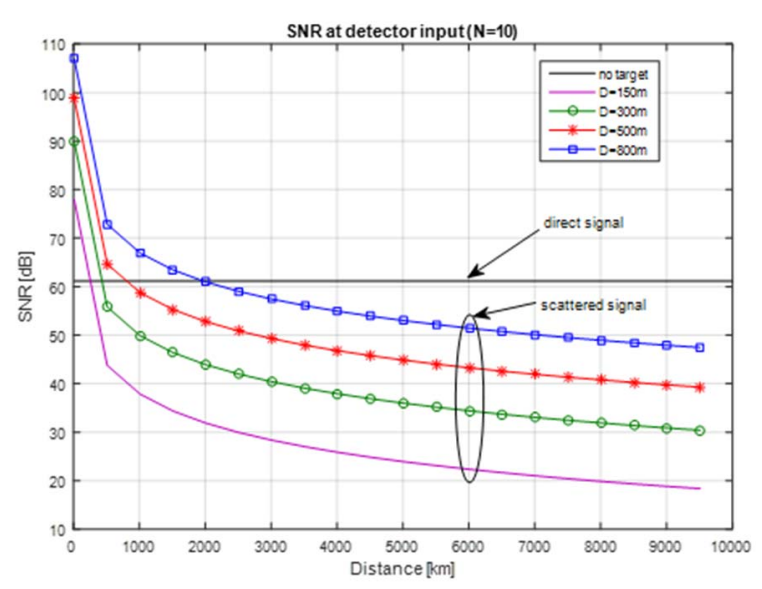

Figure 9. SNR of direct and scattered GPS signal

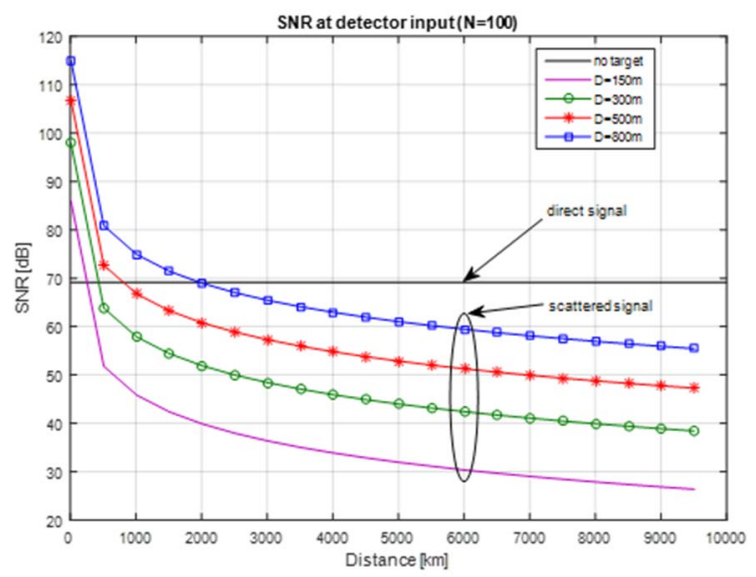

Figure11. SNR of direct and scattered GPS signal

The SNR of the re-radiated signal from cosmic objects, which depends on the number of integrations after the correlator, the diameter of the cosmic object and the distance to it, can be amplified to positive values, which allows the signal from the cosmic object by means of the subsequent processing to be separated from the direct signal and then automatically detected.

\section{Conclusions}

In the paper, we studied the dynamics in the SNR of the two signals, the reference signal and the signal from a cosmic object, at the output of the GPS FSR receiver, depending on the distance to the cosmic object and its diameter. The research also revealed the potential possibility for detection of cosmic objects depending on the distance to the object and the required amount of non-coherent integration of GPS signals.

\section{Acknowledgment}

The research work reported in the paper is supported by the project funded by Bulgarian Science Foundation, grand DFNI-T 02/3 from 12.12.2014. 


\section{References}

[1] Glennon E.P., A.G. Dempster, and C.Rizos: Feasibility of air target detection using GPS as a bistatic radar, Journal of Global Positioning Systems, vol.5, no.1-2, (2006), pp. 119-126.

[2] Clarizia M. P., P. Braca, C. Ruf, and P. Willett: Target Detection Using GPS signals of opportunity, $18^{\text {th }}$ International Conference on Information Fusion, (2015), pp. 1429-1436.

[3] Koch, V., R. Westphal: New approach to a multistatic passive radar sensor for air/space defense, IEEE AES Systems Magazine, (1995), pp. 24-32

[4] Suberviola, I., I. Mayordome, J. Mendizabal: Experimental results of air target detection with GPS forward scattering radar, IEEE Geoscience and Remote Sensing Letters, vol. 9, no. 1, (2012), pp. 47-51.

[5] Borre, K., D. Akos, N. Bertelsen, P. Rinder, S. Jensen: A Software-Defined GPS and Galileo Receiver: Single-Frequency Approach, Birkhäuser, Boston, MA, (2006).

[6] Behar, V., Chr. Kabakchiev: Detectability of Air Target Detection using Bistatic Radar Based on GPS L5 Signals, International Radar Symposium, Leipzig, Germany, (2011), pp. 212-217.

[7] Behar, V., Chr. Kabakchiev, H. Rohling: Air Target Detection Using Navigation Receivers Based on GPS L5 Signals, ION GNSS, Portland OR, (2011), pp. 333-337.

[8] Kabakchiev, C., I. Garvanov, V. Behar, H. Rohling: The Experimental Study of Possibility for Radar Target Detection in FSR Using L1-Based Non-Cooperative Transmitter, International Radar Symposium, Dresden, Germany, (2013), pp. 625-630.

[9] Kabakchiev, C., I. Garvanov, V. Behar, H. Rohling, A. Lazarov: The Experimental Study of Target FSR Shadows Detection using GPS signals, Third International Symposium on Radio Systems and Space Plasma, Sofia, Bulgaria, (2013), pp. 64-73.

[10] Kabakchie, Chr., I. Garvanov, V. Behar, P. Daskalov, H. Rohling: Moving Target FSR Shadow Detection using GPS signals, Third International Conference on Telecommunications and Remote Sensing, Luxembourg, (2014), pp. 34-40.

[11] Kabakchiev, C., V. Behar, I. Garvanov, D. Kabakchieva, L. Daniel, K. Kabakchiev, M. Gashinova, M. Cherniakov: Experimental verification of maritime target parameter evaluation in FSR, IET Radar, Sonar \& Navigation, Volume 9, Issue 4, (2015), pp. 355-363.

[12] Kabakchiev, C., V. Behar, I, Garvanov, D. Kabakchieva, H. Rohling: Detection, Parametric Imaging and Classification of Very Small Marine Targets Emerged in Heavy Sea Clutter Utilizing GPS-Based Forward Scattering Radar, The 39th International Conference on Acoustics, Speech, and Signal Processing, Florence, Italy, (2014), pp. 793-797.

[13] Kabakchiev, C., I. Garvanov, V. Behar, D. Kabakchieva, K. Kabakchiev, H.Rohling, K. Kulpa, A. Yarovoy: The Study of Target Shadows using Passive FSR Systems, International Radar Symposium, Dresden, Germany, (2015), pp. 628-633.

[14] Garvanov, I., Chr. Kabakchiev, V. Behar, M. Garvanova: Target detection using a GPS Forward-Scattering Radar. IEEE Pros. of the Second International Conference "Engineering \& Telecommunications - En\&T 2015", Moscow-Dolgoprudny, Russia, (2015), pp. 29-33. 